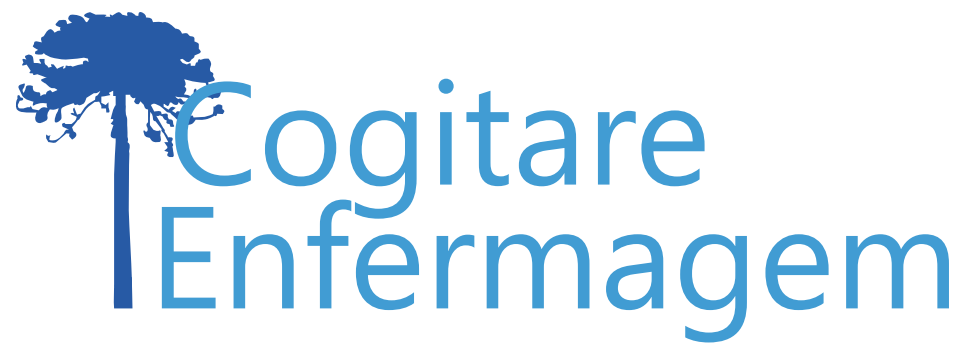

\title{
MANEJO DE MULHERES COM ATIPIAS NO EXAME CITOPATOLÓGICO DE COLO UTERINO NA ATENÇÃO PRIMÁRIA À SAÚDE*
}

Tatiana da Silva Vaz Paterra1, Priscila Alvarenga Teles ${ }^{2}$, Paola Alexandria Pinto de Magalhães ${ }^{3}$, Ana Paula Alonso Reis-Mairink ${ }^{4}$, Thais de Oliveira Gozzo ${ }^{5}$, Silvana Maria Quintana ${ }^{6}$, Marislei Sanches Panobianco ${ }^{7}$

\section{RESUMO}

Objetivo: identificar a conduta dos profissionais de saúde no manejo de mulheres com atipias no exame citopatológico, atendidas em Unidades de Atencão Primária à Saúde.

Método: estudo documental, retrospectivo, com 175 mulheres que apresentaram exames citopatológicos com atipias, entre 2006 e 2014, num município do estado de São Paulo, com análise descritiva dos dados.

Resultados: 157 (90\%) mulheres retornaram à unidade para verificar o resultado do exame. Entre as com atipias, 103 (86\%) receberam manejo adequado, porém $85(83 \%)$ repetiram o exame em tempo menor que o preconizado. Entre as que precisaram de colposcopia, $30(79 \%)$ receberam manejo adequado.

Conclusão: o manejo de mulheres com atipias foi adequado, porém o índice de positividade baixo, escassez de registros em prontuários e temporalidade inadequada para repetição do exame citopatológico demonstram um programa de rastreamento oportunístico, podendo ocasionar baixa cobertura da população alvo, alto índice de detecção tardia, e super-rastreamento.

DESCRITORES: Neoplasias do Colo do Útero; Programas de Rastreamento; Atenção Primária à Saúde; Teste de Papanicolau; Saúde da mulher.

\footnotetext{
*Artigo extraído de dissertação de mestrado "Avaliação do seguimento de mulheres com alterações no exame citopatológico do
} colo do útero". Universidade de São Paulo, 2018.

\section{COMO REFERENCIAR ESTE ARTIGO:}

Paterra T da SV, Teles PA, Magalhães PAP de, Mairink APAR, Gozzo T de O, Quintana SM, et al. Manejo de mulheres com atipias no exame citopatológico de colo uterino na atenção primária à saúde. Cogitare enferm. [Internet]. 2020 [acesso em "colocar data de acesso, dia, mês abreviado e ano"]; 25. Disponível em: http:// dx.doi.org/10.5380/ce.v25i0.66862.

Este obra está licenciado com uma Licença Creative Commons Atribuição 4.0 Internacional.

${ }^{1}$ Enfermeira. Mestre em Enfermagem. Universidade de São Paulo. Ribeirão Preto, SP, Brasil. 9 ${ }^{2}$ Enfermeira. Mestre em Enfermagem. Universidade de São Paulo. Ribeirão Preto, SP, Brasil. $(2$

${ }^{3}$ Enfermeira. Doutora em Enfermagem. Docente do Centro Universitário Padre Albino. Catanduva, SP, Brasil. $\bigcirc$

${ }^{4}$ Enfermeira. Doutora em Enfermagem em Saúde Pública. Docente do Instituto Federal de Educação, Ciência e Tecnologia do Sul de Minas Gerais. Muzambinho, MG, Brasil.

${ }^{5}$ Enfermeira. Doutora em Enfermagem. Docente de Enfermagem da Universidade de São Paulo. Ribeirão Preto, SP, Brasil.

${ }^{6}$ Médica. Doutora em Ginecologia e Obstetrícia. Docente da Universidade de São Paulo. Ribeirão Preto, SP, Brasil.

${ }^{7}$ Enfermeira. Doutora em Enfermagem. Docente de Enfermagem da Universidade de São Paulo. Ribeirão Preto, SP, Brasil. (?) 


\title{
MANAGEMENT OF WOMEN WITH ATYPIAS IN THE CERVICAL CYTOPATHOLOGICAL TEST IN PRIMARY HEALTH CARE
}

\begin{abstract}
Objective: to identify the conduct of health professionals in managing women with atypias in the cervical cytopathological test who received care in primary healthcare units.

Methods: documentary and retrospective study carried out with 175 women who had atypias in their cervical cytopathological tests between 2006 and 2014 in a municipality in the state of São Paulo, Brazil, with descriptive analysis of collected data.

Results: 157 (90\%) women went back to the unit to check the test result. Among those with atypias, 103 (86\%) were properly managed, but 85 (83\%) repeated the test within a period shorter than the advocated one. In the group of women who needed a colposcopy, 30 (79\%) were properly managed.

Conclusion: the management of women with atypias was adequate, but the low positivity rate, the lack of patient records, and the inadequate time to repeat the cervical cytopathological test indicated an opportunistic screening program, which may lead to low coverage of the target population, high late detection rate, and overscreening.
\end{abstract}

DESCRIPTORS: Uterine Cervical Neoplasms; Mass Screening; Primary Health Care; Papanicolaou Test; Women's Health.

\section{MANEJO DE MUJERES CON ATIPIAS EN ESTUDIO CITOPATOLÓGICO DE CUELLO UTERINO EN ATENCIÓN PRIMARIA}

\begin{abstract}
RESUMEN:
Objetivo: Determinar la conducta de los profesionales de salud para manejo de mujeres con atipias en examen citopatológico, atendidas en Unidades de Atención Primaria de Salud.

Método: Estudio documental, retrospectivo, con 175 mujeres con estudios citopatológicos mostrando atipias, entre 2006 y 2014, en municipio del estado de São Paulo, con análisis descriptivo de datos.

Resultados: 157 (90\%) mujeres volvieron a la unidad para verificar el resultado del estudio. Entre las que presentaron atipias, 103 (86\%) recibieron manejo adecuado, aunque 85 (83\%) repitieron el estudio en tiempo inferior al recomendado. De las que precisaron colposcopia, $30(79 \%)$ recibieron manejo adecuado.

Conclusión: El manejo de mujeres con atipias resultó adecuado, aunque el bajo índice de positividad, escasez de registros en historias clínicas y temporalidad inadecuada de reiteración del estudio demuestran programación oportuna del seguimiento, determinando potencial baja cobertura de la población objetivo, alto índice de detección tardía y sobreseguimiento.
\end{abstract}

DESCRIPTORES: Neoplasias del Cuello Uterino; Tamizaje Masivo; Atención Primaria de Salud; Prueba de Papanicolau; Salud de la Mujer. 
No Brasil, o controle do câncer de colo uterino (CCU) constitui uma das prioridades da agenda de saúde. Apesar das iniciativas governamentais direcionadas à sua prevenção e controle, o país apresenta elevada incidência e mortalidade por essa doença, que em 2017 foi responsável por 6.385 mortes, ocupando a terceira posição dentre as neoplasias malignas na mulher ${ }^{(1,2)}$.

O Instituto Nacional de Câncer José Alencar Gomes da Silva (INCA) estima 16.370 casos novos da doença para cada ano do biênio 2018-2019, e a implementação de programas efetivos de rastreamento das lesões pré-neoplásicas ou invasoras em estágio inicial tem sido apontada como uma das formas de redução dessas taxas ${ }^{(1,2)}$. Neste sentido, o Ministério da Saúde (MS) recomenda a realização do exame citopatológico como estratégia primordial para o rastreamento do CCU e lesões precursoras, visto que a doença detectada em fases iniciais tem alto índice de cura ${ }^{(3-5)}$.

Desde 2011, com a publicação das Diretrizes Brasileiras para o Rastreamento do Câncer do Colo do Útero, os profissionais de saúde têm padronizado as condutas para o manejo das atipias celulares, além da uniformização da oferta do exame citopatológico para mulheres com idade entre 25 e 64 anos que já iniciaram atividade sexual, após realização de dois exames anuais, consecutivos, com resultado normal, repetir a cada três anos ${ }^{(6)}$.

A partir de exame citopatológico com resultado alterado, ou seja, apresentando atipias celulares, é necessário assegurar o adequado manejo da mulher, com garantia de percurso assistencial articulado, acesso aos serviços de saúde e cuidado integral. Caso haja inadequação desse manejo, a mulher corre o risco de atraso no diagnóstico, com perda de oportunidade de identificação na fase de lesão precursora ou câncer inicial(7).

A Atenção Primária à Saúde (APS) se constitui na porta de entrada do usuário no sistema de saúde, sendo responsável por atividades de prevenção do CCU, vacinação contra o HPV (Papiloma Vírus Humano) e detecção precoce da doença ou de suas lesões precursoras. Os profissionais atuantes nesse nível de atenção devem conhecer o método, a periodicidade e a população-alvo recomendados para o rastreio do CCU, sabendo ainda orientar e encaminhar as mulheres para tratamento de acordo com os resultados dos exames, além de garantir seu seguimento ${ }^{(8,9)}$.

A proposta deste estudo se embasou em informações da literatura, tanto nacional quanto internacional, que apontam a pouca observância das diretrizes do programa de rastreamento do CCU preconizadas pelos órgãos competentes de atenção à saúde da mulher de alguns países, em especial aqueles em desenvolvimento. Evidenciam que as mulheres com exames alterados receberam conduta de encaminhamento inadequada e que os serviços de saúde não estão articulados, o que resulta em importantes falhas no seguimento ${ }^{(5,7,10-12)}$. Além disso, no Brasil, as taxas de cobertura de rastreio do CCU estão distantes do preconizado pelo MS, que é de 80 a $85 \%{ }^{(4)}$.

Dessa forma, o objetivo deste estudo foi identificar a conduta dos profissionais de saúde no manejo de mulheres com atipias no exame citopatológico, atendidas em Unidades de Atenção Primária à Saúde.

\section{MÉTODO}

Estudo retrospectivo, descritivo, documental realizado em nove unidades de saúde da APS em um município do interior do estado de São Paulo.

A coleta de dados foi realizada no período de maio a agosto de 2017 e direcionada por um instrumento contendo as seguintes variáveis: perfil sociodemográfico (idade, 
escolaridade, estado civil e cor) e relativas ao manejo da mulher na APS (conduta dos profissionais de saúde frente ao resultado de exame com atipias, intervalos de tempo entre a coleta do exame com atipias e o retorno para verificação do resultado, tempo para repetição do exame, tempo médio entre o encaminhamento da APS e realização de colposcopia no serviço de referência).

Inicialmente, fez-se um levantamento, consultando o Sistema de Informação do Câncer do Colo do Útero (SISCOLO) e Sistema de Informação do Câncer (SISCAN̉) para identificar mulheres com exames citopatológicos apresentando atipias. Foi observado que algumas delas tinham vários resultados evidenciando atipias celulares. Foi escolhido o primeiro exame citopatológico com atipias, no período em estudo, para avaliação da conduta dos profissionais.

Posteriormente, foi realizada visita às unidades da APS a fim de obter nos prontuários informações relativas às condutas dos profissionais de saúde. O município conta com o apoio de serviço de referência para os casos que necessitam de colposcopia, investigação diagnóstica e tratamento.

Para aquelas encaminhadas para o serviço de referência para realização de colposcopia, investigação diagnóstica e tratamento, também foi realizada consulta aos prontuários do serviço para obtenção de dados complementares.

Os profissionais que atuavam no manejo de mulheres com atipias no exame citopatológico no município em que ocorreu a coleta de dados eram: médico ginecologista, enfermeiro e agente comunitário de saúde. O enfermeiro registrava no prontuário o resultado do exame e realizava a busca ativa com auxílio do agente comunitário de saúde. A colposcopia, quando necessário, era solicitada pelo médico ginecologista.

Foram identificados os resultados de 25.366 exames citopatológicos entre janeiro de 2006 e dezembro de 2014 na APS, sendo que $24.972(98,5 \%)$ foram classificados como negativos, e $394(1,5 \%)$ apresentaram atipias celulares (Figura 1).

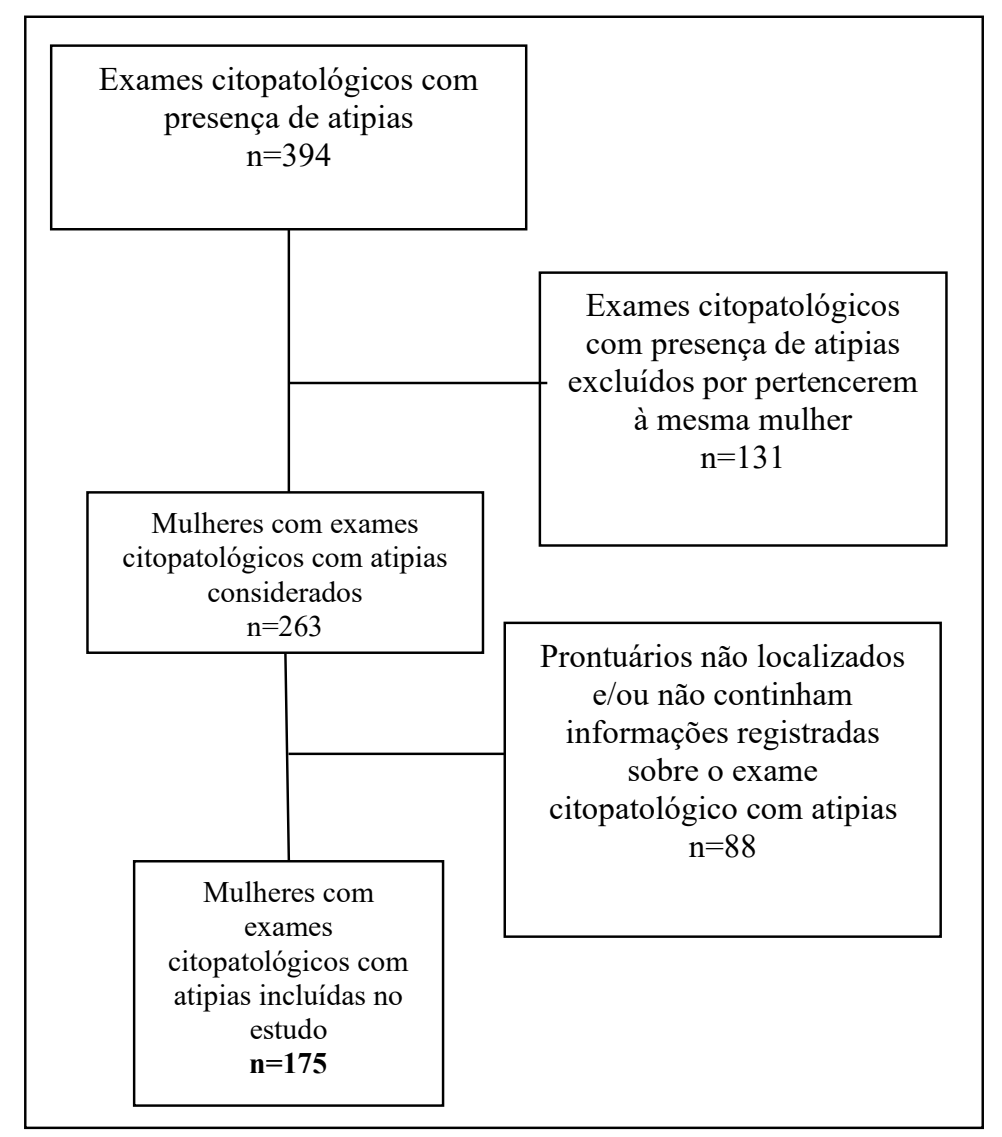

Figura 1 - Fluxograma da composição da amostra do estudo. Batatais, SP, Brasil, 2017 
A partir destes resultados, foram excluídos exames de mulheres cujo prontuário não foi possível localizar ou aqueles que não possuíam registros de informações sobre exame citopatológico alterado. Com isso, foram incluídas 175 mulheres com exame citopatológico que apresentaram atipia celular no resultado, conforme Figura 1.

Para categorizar os resultados de exames com atipias celulares, foi utilizada a Nomenclatura Brasileira para Laudos Cervicais ${ }^{(13)}$. Para avaliar a conduta dos profissionais da saúde no manejo das atipias, utilizou-se a Diretriz Nacional de Rastreamento do Câncer do Colo do Útero, vigente na data da coleta do exame ${ }^{(14,15)}$.

Os dados foram armazenados em planilha de Excel 2010, com dupla digitação e validação. $\mathrm{Na}$ análise descritiva dos dados, foi realizada a distribuição percentual das variáveis categóricas e, para as variáveis contínuas, medidas de tendência central e dispersão.

Atendendo à Resolução do Conselho Nacional de Saúde (CNS) n 466, de 12 de dezembro de 2012, o estudo foi aprovado por Comitê de Ética em Pesquisa com Seres Humanos (CEP) sob parecer 2.000.927(16).

\section{RESULTADOS}

Foram analisados os prontuários de 175 mulheres com resultado citopatológico de atipias. A média de idade das mulheres estudadas foi de 43 anos $( \pm 13,79)$, sendo que 132 (75,43\%) estavam na faixa etária da população alvo de 25 a 64 anos, 39 (22,29\%) estavam abaixo dos 25 anos, e quatro (2,28\%) acima dos 64 anos. A maioria 145 (82,86\%) era branca e $154(88 \%)$ não possuíam informações registradas em prontuário sobre estado civil e 161 (92\%) de escolaridade.

Dentre as 175 mulheres estudadas, observou-se que 157 (90\%) retornaram à unidade de saúde para verificação do resultado do exame citopatológico, com mediana de intervalo de tempo entre coleta do exame e verificação do resultado de 42 dias. Em relação às demais mulheres com atipias, em $18(10 \%)$ não havia registro no prontuário a respeito de retorno para verificação do resultado do exame ou busca ativa. As mulheres das quais não foi possivel avaliar o manejo $18(10 \%)$, apresentavam as seguintes atipias: ASC-US $(n=10)$, LSIL $(n=6)$ e HSIL $(n=2)$. Portanto, foi possível avaliar a conduta dos profissionais de saúde da APS de 157 (90\%) mulheres com atipias.

Das 157 mulheres com exame citopatológico apresentando atipias celulares, foi possível observar que 119 (76\%) diziam respeito a dois tipos de atipias: ASC-US e LSIL (Tabela 1).

Tabela 1 - Resultados dos exames citopatológicos de mulheres com atipias celulares $(n=157)$ atendidas em município do interior do estado de São Paulo. Brasil, 2017 (continua)

\begin{tabular}{lcc} 
Atipias celulares & n & $\%$ \\
\hline ASC-US & 57 & 38,28 \\
\hline ASC-H & 4 & 2,29 \\
\hline AGC-US & 2 & 2,29 \\
\hline AGC-H & 3 & 1,71 \\
\hline AOI-II & 1 & 0,57 \\
\hline
\end{tabular}




\begin{tabular}{lcc}
\hline LSIL & 62 & 38,86 \\
\hline HSIL & 17 & 10,86 \\
\hline HSIL microinvasão & 4 & 2,29 \\
\hline Carcinoma & 5 & 2,85 \\
\hline AIS & 2 & 2,29 \\
\hline Total & 157 & 100
\end{tabular}

Legenda: Células escamosas atípicas de significado indeterminado (ASC) possivelmente não neoplásicas (ASC-US) ou não podendo excluir lesão intraepitelial de alto grau (ASC-H); células glandulares atípicas de significado indeterminado (AGC), possivelmente não neoplásicas (AGC-US) ou não se pode excluir lesão intraepitelial de alto grau (AGC-H); células atípicas de origem indefinida que não se pode excluir lesão intraepitelial de alto grau (AOI-II); lesão intraepitelial de baixo grau (LSIL), lesão intraepitelial de alto grau (HSIL), HSIL não podendo excluir microinvasão (HSIL microinvasão), adenocarcinoma in situ (AIS)(14).

Para as 119 mulheres com resultado ASC-US ( $n=57)$ e LSIL $(n=62)$, que tiveram seguimento no serviço de saúde, observou-se que $86 \%$ tiveram o manejo adequado, cuja recomendação é a repetição do exame $(17,18)$. Entre aquelas para as quais foi recomendado repetir o exame citopatológico, 83\% compareceram para realização da citologia. Entre as 62 mulheres com resultado LSIL e que repetiram o exame, $79 \%$ coletaram a citologia em menos de seis meses, ou seja, tempo menor que o recomendado pelas diretrizes de rastreamento do CCU(14,15). Para as 57 mulheres com resultado ASC-US, 65\% repetiram o exame e $57 \%$ delas realizou a coleta em tempo menor que o recomendado.

Para as 38 mulheres com resultados ASC-H, AGC-US, AGC-H, AOI-II, HSIL, AIS, HSIL microinvasor e carcinoma, que tiveram seguimento no serviço de saúde, observou-se que $79 \%$ tiveram o manejo adequado, de acordo com as diretrizes brasileiras que preconiza solicitação de colposcopia ${ }^{(13,14)}$ (Figura 2 ) e $83 \%$ realizaram o exame. A mediana do intervalo de tempo entre a data da solicitação e realização da colposcopia foi de 40 dias. 


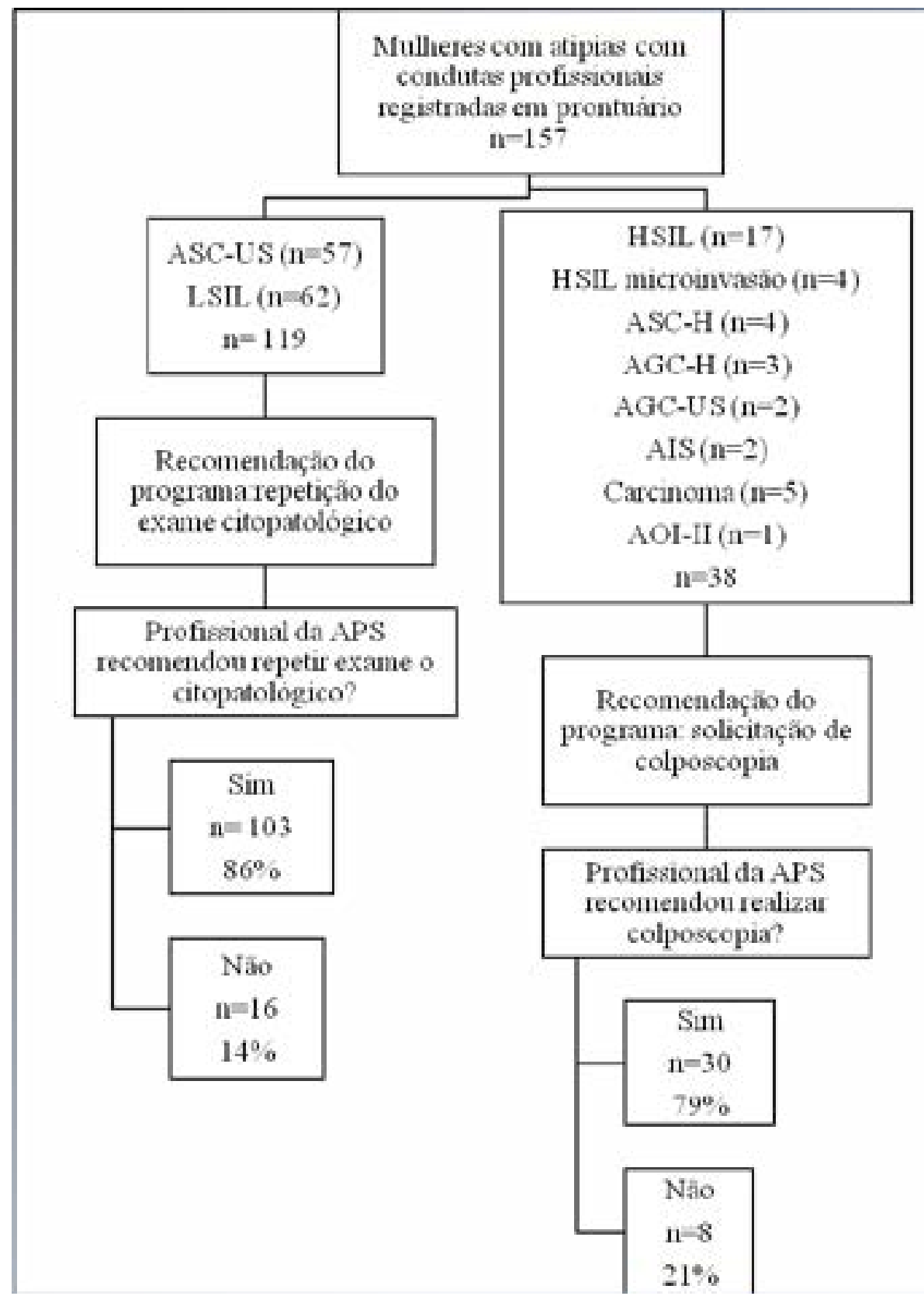

Figura 2- Conduta dos profissionais de saúde no manejo de mulheres com atipias celulares atendidas em município do interior do estado de São Paulo. Batatais, SP, Brasil, 2017

Para as 38 mulheres encaminhadas para o serviço de referência para realização de colposcopia, seguimento e/ou tratamento, foi verificado que o tempo entre o encaminhamento da APS e a primeira consulta no serviço apresentou mediana de 21 dias.

Ao término da coleta dos dados, das 38 mulheres encaminhadas para o serviço de referência, $50 \%$ estavam em acompanhamento ginecológico regular na APS; $20 \%$ frequentavam a APS, mas não estavam em acompanhamento ginecológico; $15 \%$ não estavam frequentando a unidade de saúde; de uma não foi localizado registro sobre o último atendimento na APS e cinco foram a óbito. As causas dos óbitos de acordo com a classificação internacional de doenças $\left(\right.$ CID-10) ${ }^{(16)}$ foram: neoplasia maligna do útero (1), lesão invasiva dos órgãos genitais femininos (1), neoplasia maligna do trato intestinal, parte não especificada (1), outras formas de obstrução intestinal (1) e causas mal definidas (1). 
O acompanhamento dos percentuais de exames alterados é denominado índice de positividade, e o MS recomenda que ele esteja entre 3\% e 10\%. Esse valor expressa o percentual de exames com resultados alterados em relação ao total de exames realizados no mesmo local e período. Avalia também a sensibilidade do processo de rastreamento para detectar lesões na população ${ }^{(18)}$.

Neste estudo foi observado um índice de positividade baixo [394 (1,5\%)], indicando que alterações suspeitas podem não ter sido identificadas pelo laboratório e ter acarretado exames falsos-negativos. Em relação ao percentual de alterações, pesquisas realizadas em outros municípios brasileiros mostraram uma variação de $1,02 \%{ }^{(17)}$ na região sul a $7,37 \%$ na região centro-oeste ${ }^{(5)}$. Nesse sentido, ressalta-se a importância da coleta do exame citopatológico de maneira adequada, seguindo todas as etapas recomendadas: checar se a mulher realizou o preparo conforme preconizado, identificação da lâmina, coleta dupla, preparo do esfregaço, fixação do material e acondicionamento adequado. Assim, a qualidade do exame está diretamente relacionada ao sucesso do programa de rastreamento do $\mathrm{CCU}^{(19)}$.

Observou-se ainda que a falta das informações registradas nos prontuários dificultou a obtenção de dados, impossibilitando delinear um perfil sociodemográfico das mulheres, especialmente o estado civil e a escolaridade. Essas informações são importantes, uma vez que a escolaridade constitui fator de risco para desenvolvimento do CCU, ou seja, quanto menor o grau de escolaridade, maior a chance de ocorrência do agravo(20). A inconformidade da anotação, quer pela ausência de registro ou incompletude, dificulta a comunicação eficaz entre os profissionais e a continuidade das ações assistenciais ${ }^{(21)}$.

Em relação à raça/cor, houve prevalência da cor branca, sendo um dado esperado, pois segundo o último censo do município, o número de mulheres brancas de 25 a 64 anos era de 11.508, enquanto o de não brancas, no mesmo período e faixa etária, era de $3.733^{(22)}$.

Em relação à população-alvo, a maioria das mulheres desse estudo estava na faixa etária preconizada para o rastreamento. O citopatológico em mulheres entre 35 e 64 anos é muito mais efetivo em detectar lesões progressivas do que em mulheres por volta dos 20 $\operatorname{anos}^{(23)}$ e, ainda, o rastreamento em mulheres com menos de 25 anos não tem impacto na redução da incidência e mortalidade por CCU. Estudo realizado na Inglaterra evidenciou que o rastreamento anterior aos 25 anos não tem impacto importante na redução do CCU, pois é raro esse tipo de câncer na faixa etária entre 20 a 24 anos $^{(24)}$. Além disso, elevado percentual de lesões intraepiteliais regride espontaneamente antes dos 25 anos ${ }^{(25)}$.

Para as mulheres com alterações, cuja recomendação do programa é indicação de colposcopia, foi observado que a mediana do intervalo de tempo entre a data de solicitação e realização de colposcopia foi de 40 dias. Nas diretrizes brasileiras de rastreamento do $\mathrm{CCU}^{(14,15)}$, não há menção sobre a temporalidade adequada referente à realização da colposcopia e, assim, foram utilizadas como base as diretrizes europeias de rastreamento do $\operatorname{CCU}^{(23)}$ para avaliar a adequabilidade do tempo neste estudo: para lesões de alto grau, não deve ser superior a quatro semanas. Dessa forma, o tempo de realização da colposcopia encontrado neste estudo esteve próximo do recomendado. Estudo realizado no Rio de Janeiro-RJ, na baixada fluminense, com 1227 mulheres, avaliou o tempo de espera para obter a primeira colposcopia por mulheres com citopatológico alterado. Foi verificado que $71,69 \%$ das mulheres tiveram acesso ao exame dentro de 30 ou de 60 dias a partir da data do encaminhamento da unidade de origem ${ }^{(26)}$.

O intervalo de tempo entre a coleta do exame citopatológico e o retorno da paciente ao serviço para a comunicação do resultado ficou em torno de 42 dias, intervalo adequado, pois o recomendado é que seja de dez dias para envio da lâmina ao laboratório, e até 30 dias para o laboratório emitir o resultado(4).

Apesar de $86 \%$ das mulheres com ASC-US e LSIL, apresentarem o manejo adequado, com a repetição do exame, a temporalidade de repetição foi inadequada para $36 \%$ delas, pois a realização do exame ocorreu antes de seis meses, independentemente da idade. $O$ 
exame citopatológico não deveria ser repetido com intervalo menor do que o preconizado, uma vez que o epitélio cervical precisa de um tempo para regenerar ${ }^{(22)}$; e $55 \%$ e $67 \%$ das infecções por HPV carcinogênicos regridem espontaneamente em seis e 12 meses, respectivamente ${ }^{(27)}$.

Em relação à conduta imediata dos profissionais da APS frente aos resultados ASC-H, AGC-US, AGC-H, AOI-II, HSIL e HSIL não podendo excluir microinvasão, verificou-se que $79 \%$ das mulheres tiveram manejo adequado, isto é, foi solicitada a colposcopia $a^{(11,15)}$. Resultado diferente foi encontrado em estudo realizado no município de Goiânia-GO, no período de 2006 a 2008, que observou que $61,22 \%$ das mulheres com exame citopatológico alterado tiveram seguimento ignorado e, que possivelmente, não foram submetidas às condutas preconizadas pelo MS para rastreamento de $\mathrm{CCU}^{(5)}$.

A mediana do intervalo de tempo entre a data do encaminhamento e a primeira consulta no serviço de referência foi de 21 dias, tempo adequado, considerando que a mulher tem o direito de iniciar o tratamento em até 60 dias após o diagnóstico de câncer ${ }^{(28)}$.

Em relação às mulheres que perderam seguimento nas diferentes etapas, foi observada uma quantidade reduzida dos registros de busca ativa, o que pode influenciar de maneira negativa a eficácia do Programa de Rastreamento. A busca ativa deve ser realizada para garantir o diagnóstico precoce e melhora do prognóstico do CCU, assim como para impactar positivamente na morbimortalidade por esse tipo de câncer.

Ao fim deste estudo, os dados confirmaram que cinco mulheres foram a óbito, e apenas uma delas teve o CCU registrado como causa do óbito. Para as demais mulheres, as causas sugerem uma correlação do óbito com o CCU.

Algumas características como índice de positividade baixo, alta adesão das mulheres seguidas, tempo inadequado de repetição do exame e escassez de registros sobre busca ativa sugerem que o programa de rastreamento avaliado é ainda oportunístico. Este tipo de rastreamento consiste na prática de coletar exame citopatológico pela iniciativa própria da mulher ou em qualquer oportunidade, por exemplo, consulta clínica para outros propósitos ${ }^{(23)}$. Tal ação, por vezes, está em desacordo com as diretrizes de rastreamento estabelecidas e pode ocasionar baixa cobertura da população alvo, alto índice de detecção tardia, e super-rastreamento de determinados grupos.

Pode-se considerar como limitações do estudo a falta de informações de exames realizados na atenção suplementar/privada, resultados de exames citopatológicos anteriores ao alterado, e as consequências das falhas nas condutas de encaminhamento. Ressalta-se que a baixa qualidade da informação obtida de fontes secundárias, principalmente dos prontuários, dificultou a obtenção de dados.

\section{CONCLUSÃO}

A conduta dos profissionais de saúde no manejo de mulheres com atipias no exame citopatológico, atendidas em Unidades da APS, foi considerada adequada para a maioria dos casos avaliados, assim como o tempo gasto nas diferentes etapas: tempo entre a coleta do exame citopatológico e a comunicação do resultado à mulher; tempo entre o encaminhamento e a primeira consulta no serviço de referência.

No entanto, algumas características, como índice de positividade baixo, escassez de registros em prontuários e temporalidade inadequada para repetição do exame citopatológico, sugerem que o programa de rastreamento ainda é oportunístico. Assim, evidenciaram-se alguns aspectos do manejo dessas mulheres que podem ser melhorados, visando, principalmente, à redução de iniquidades de acesso e ao incremento do diagnóstico precoce do CCU, favorecendo o prognóstico clínico delas. 
Este trabalho de campo evidenciou dificuldades de diferentes responsabilidades no âmbito de implementação e de gestão. Os dados apresentados podem contribuir para a tomada de decisão de coordenadores de unidades de saúde, assim como para os profissionais nelas inseridos, visando ao incremento do diagnóstico precoce do CCU e favorecimento do bom prognóstico clínico dessas mulheres.

\section{REFERÊNCIAS}

1. Vale DBAP do, Morais SS, Pimenta AL, Zeferino LC. Avaliação do rastreamento do câncer do colo do útero na Estratégia Saúde da Família no Município de Amparo, São Paulo, Brasil. Cad. Saúde Pública. [Internet]. 2010 [acesso em 28 out 2017]; 26(2). Disponível em: http://dx.doi.org/10.1590/S0102311X2010000200017.

2. Navarro C, Fonseca AJ da, Sibajev A, Souza Cl de A, Araújo DS, Teles DA de F, et al. Cobertura do rastreamento do câncer de colo de útero em região de alta incidência. Rev. Saúde Públ. [Internet]. 2015 [acesso em 19 jan 2018]; 49(17). Disponível em: http://dx.doi.org/10.1590/S0034-8910.2015049005554.

3. Arzuaga-Salazar MA, Souza M de L de, Martins HEL, Locks MTR, Monticelli M, Peixoto HG. Câncer de colo do útero: mortalidade em Santa Catarina - Brasil, 2000 a 2009. Texto contexto-enferm.

[Internet]. 2011 [acesso em 17 nov 2017]; 20(3). Disponível em: http://dx.doi.org/10.1590/S0104$\underline{07072011000300016 .}$

4. Ministério da Saúde (BR). Secretaria de Atenção à Saúde. Departamento de Atenção Básica. Controle dos cânceres do colo do útero e da mama. [Internet] Brasília: Ministério da Saúde; 2013 [acesso em 05 jan 2017]. Disponível em: http://bvsms.saude.gov.br/bvs/publicacoes/controle canceres colo utero 2013. pdf.

5. Araújo ES, Barbosa FM, Ázara CZS, Ferreira TXAM, Tavares SB do N, Amaral RG. Avaliação do seguimento de mulheres com exames citopatológicos alterados de acordo com as condutas preconizadas pelo Ministério da Saúde do Brasil em Goiânia, Goiás. Rev. bras. cancerol. [Internet]. 2014 [acesso em 16 dez 2017]; 60(1). Disponível em: https://rbc.inca.gov.br/site/arquivos/n 60/v01/pdf/03-artigo-avaliacaodo-seguimento-de-mulheres-com-exames-citopatologicos-alterados-de-acordo-com-as-condutaspreconizadas-pelo-ministerio-da-saude-do-brasil-em-goiania-goias.pdf.

6. Ministério da Saúde (BR). Instituto Nacional do Câncer José Alencar Gomes da Silva (INCA). Diretrizes brasileiras para o rastreamento do câncer do colo do útero. 2. ed. [Internet] Rio de Janeiro: Ministério da Saúde; 2016 [acesso em 19 mar 2018]. Disponível em: http://www.citologiaclinica.org.br/site/pdf/ documentos/diretrizes-para-o-rastreamento-do-cancer-do-colo-do-utero 2016.pdf.

7. Albuquerque ZBP de, Manrique EJC, Tavares SB do N, Souza ACS e, Guimarães JV, Amaral RG. Mulheres com atipias, lesões precursoras e invasivas do colo do útero: condutas segundo recomendações do Ministério da Saúde. Rev Bras Ginecol Obstet. [Internet]. 2012 [acesso em 13 mar 2018]; 34(6). Disponível em: http://www.scielo.br/pdf/rbgo/v34n6/v34n6a02.pdf.

8. Ministério da Saúde (BR). Instituto Nacional do Câncer José Alencar Gomes da Silva (INCA). Agenda estratégica para o controle do câncer do colo do útero. [Internet] Rio de Janeiro: Ministério da Saúde; 2011 [acesso em 23 ago 2015]. Disponível em: https://www.inca.gov.br/utero.

9. Ministério da Saúde (BR). Secretaria de Atenção à Saúde. Departamento de Atenção Básica. Controle dos cânceres do colo do útero e da mama. [Internet] Brasília: INCA; 2013 [acesso em 20 ago 2016]. Disponível em: http://bvsms.saude.gov.br/bvs/publicacoes/controle canceres colo utero 2013.pdf.

10. Wiesner C, Cendales R, Murillo R, Piñeros M, Tovar S. Seguimiento de mujeres con anormalidad citológica de cuello uterino, en Colombia. Rev. salud pública. [Internet]. 2010 [acesso em 13 abr 2018]; 12(1). Disponível em: https://scielosp.org/pdf/rsap/2010.v12n1/1-13/es.

11. Farias ACB de, Barbieri AR. Seguimento do câncer de colo de útero: estudo da continuidade da assistência à paciente em uma região de saúde. Esc. Anna Nery. [Internet]. 
2016 [acesso em 04 jan 2017]; 20(4). Disponível em: http://www.scielo.br/scielo.php?script=sci arttext\&pid=S1414-81452016000400213.

12. Kupets $R$, Lu Y, Vicus D, Paszat L. Are there flaws in the follow-up of women with low-grade cervical dysplasia in Ontario? J Obstet Gynaecol Can [Internet]. 2014 [acesso em 08 fev 2018]; 36(10). Disponível em: https://doi.org/10.1016/S1701-2163(15)30438-2.

13. Ministério da Saúde (BR). Secretaria de Gestão Estratégica e Participativa. Departamento de Articulação Interfederativa. SISPACTO. [Internet]. Brasília: Ministério da Saúde; 2015 [acesso em 15 mar 2018]. Disponível em: http://aplicacao.saude.gov.br/sispacto.

14. Ministério da Saúde (BR). Instituto Nacional do Câncer José Alencar Gomes da Silva (INCA). Nomenclatura brasileira para laudos cervicais e condutas preconizadas: recomendações para profissionais de saúde. [Internet]. Rio de Janeiro: Ministério da Saúde; 2006 [acesso em 18 mar 2018]. Disponível em: http://bvsms.saude.gov.br/bvs/publicacoes/Nomenclaturas 2 1705.pdf.

15. Ministério da Saúde (BR). Instituto Nacional do Câncer José Alencar Gomes da Silva (INCA). Diretrizes brasileiras para o rastreamento do câncer do colo do útero. [Internet]. Rio de Janeiro: Ministério da Saúde; 2011 [acesso em 25 mar 2018]. Disponível em: http://bvsms.saude.gov.br/bvs/publicacoes/inca/ rastreamento cancer colo utero.pdf.

16. Ministério da Saúde (BR). Conselho Nacional de Saúde. Diretrizes e normas regulamentadoras de pesquisa envolvendo seres humanos. Resolução n. 466, de 12 de dezembro de 2012. Brasília: Ministério da Saúde; 2012.

17. Organização Mundial da Saúde (OMS). CID -10: classificação estatística internacional de doenças e problemas relacionados à saúde. 10. ed. São Paulo: Universidade de São Paulo; 1997.

18. Ministério da Saúde (BR). Instituto Nacional do Câncer José Alencar Gomes da Silva (INCA). Coordenação Geral de Prevenção e Vigilância. Divisão de Detecção Precoce e Apoio à Organização de Rede. Ficha técnica de indicadores das ações de controle do câncer do colo do útero. [Internet]. Rio de Janeiro: Ministério da Saúde; 2014 [acesso em 18 mar 2018]. Disponível em: https://www.inca.gov.br/ sites/ufu.sti.inca.local/files//media/document//fichatecnicaindicadorescolo14.pdf.

19. Ministério da Saúde (BR). Instituto Nacional do Câncer José Alencar Gomes da Silva (INCA). Monitoramento das ações de controle dos cânceres do colo do útero e de mama. [Internet]. Rio de Janeiro: INCA; 2015 [acesso em 02 ago 2016]. Disponível em: https://www.inca.gov.br/sites/ufu.sti.inca. local/files/media/document/informativo-deteccao-precoce-1-2015.pdf.

20. Mascarello KC, Silva NF, Piske MT, Viana KCG, Zandonade E, Amorim MHC. Perfil sociodemográfico e clínico de mulheres com câncer do colo do útero associado ao estadiamento inicial. Rev. bras. cancerol. [Internet]. 2012 [acesso em 28 jan 2018]; 58(3). Disponível em: http://www1.inca.gov.br/rbc/n 58/v03/ pdf/11 artigo perfil sociodemografico clinico mulheres cancer colo utero associado estadiamento inicial.pdf.

21. Borges FFD, Azevedo CT de, Amorim TV, Figueiredo MAG, Ribeiro RGM. Importância das anotações de enfermagem segundo a equipe de enfermagem: implicações profissionais e institucionais. Rev. enferm. Cent.-Oeste Min. [Internet]. 2017 [acesso em 14 jan 2018]; 7:e1147. Disponível em: http://dx.doi. org/10.19175/recom.v7i0.1147.

22. Instituto Brasileiro de Geografia e Estatística (IBGE). Censo 2010. [Internet]. Rio de Janeiro: IBGE; 2010 [acesso em 19 fev 2018]. Disponível em: https://censo2010.ibge.gov.br/.

23. Arbyn M, Anttila A, Jordan J, Ronco G, Schenck U, Segnan N, et al. European Guidelines for Quality Assurance in Cervical Cancer Screening. Second Edition - Summary Document. Ann. Oncol. [Internet]. 2010 [acesso em 16 dez 2017]; 21(3). Disponível em: https://doi.org/10.1093/annonc/mdp471.

24. Castanon A, Leung VMW, Landy R, Lim AWW, Sasieni P. Characteristics and screening history of women diagnosed with cervical cancer aged 20-29 years. Br J Cancer [Internet]. 2013 [acesso em 19 set 2017]; 109(1). Disponível em: https://doi.org/10.1038/bjc.2013.322. 
25. Ministério da Saúde (BR). Instituto Nacional do Câncer José Alencar Gomes da Silva (INCA). Controle do câncer do colo do útero: detecção precoce. [Internet]. Rio de Janeiro: INCA; 2018 [acesso em 13 abr 2018]. Disponível em: https://www.inca.gov.br/controle-do-cancer-do-colo-do-utero/acoes-de-controle/

deteccao-precoce.

26. Nascimento GW de C, Pereira CC de A, Nascimento DI de C, Lourenço GC, Machado CJ. Cobertura do exame citopatológico do colo do útero no estado de Minas Gerais, Brasil, no período entre 2000-2010: um estudo a partir dos dados do Sistema de Informação do Câncer do Colo do Útero (SISCOLO). Cad. Saúde Colet. [Internet]. 2015 [acesso em 17 set 2017]; 23(3). Disponível em: https://doi. org/10.1590/1414-462X201500030059.

27. Rodríguez AC, Schiffman M, Herrero R, Wacholder S, Hildesheim A, Castle PE, et al. Rapid clearance of human papillomavirus and implications for clinical focus on persistent infections. J. Natl. Cancer Inst. [Internet]. 2008 [acesso em 18 jan 2018]; 100(7). Disponível em: https://doi.org/10.1093/jnci/djn044.

28. Brasil. Lei n. 12.732, de 22 de novembro de 2012. Dispõe sobre o primeiro tratamento de paciente com neoplasia maligna comprovada e estabelece prazo para seu início. Diário Oficial da União. [Internet]. Brasília, 23 nov. 2012 [acesso em 13 maio 2018]. Disponível em: http://www.planalto.gov.br/ccivil 03/ ato2011-2014/2012/lei/l12732.htm.

Recebido: 14/05/2019

Finalizado: $18 / 03 / 2020$

Autor Correspondente:

Marislei Sanches Panobianco

Universidade de São Paulo

Av. Bandeirantes, 3900 - 14040-902 - Ribeirão Preto, SP, Brasil

E-mail: marislei@eerp.usp.br

Contribuição dos autores:

Contribuições substanciais para a concepção ou desenho do estudo; ou a aquisição, análise ou interpretação de dados do estudo - TSVP, APARM, TOG, MSP

Elaboração e revisão crítica do conteúdo intelectual do estudo - PAT, PAPM, SMQ

Aprovação da versão final do estudo a ser publicado - APARM, TOG, MSP 